\title{
Recombining Trinomial Tree for Real Option Valuation with Changing Volatility
}

\author{
Tero Haahtela \\ Helsinki University of Technology, P.O. Box 5500, 02015 TKK, Finland \\ +358505771690 tero.haahtela@tkk.fi
}

\begin{abstract}
This paper presents a recombining trinomial tree for valuing real options with changing volatility. The trinomial tree presented in this paper is constructed by simultaneously choosing such a parameterization that sets a judicious state space while having sensible transition probabilities between the nodes. The volatility changes are modeled with the changing transition probabilities while the state space of the trinomial tree is regular and has a fixed number of time and underlying asset price levels. The presented trinomial lattice can be extended to follow a displaced diffusion process with changing volatility, allowing also taking into account the level of the underlying asset price. The lattice can also be easily parameterized based on a cash flow simulation, using ordinary least squares regression method for volatility estimation. Therefore, the presented recombining trinomial tree with changing volatility is more flexible and robust for practice use than common lattice models while maintaining their intuitive appeal.
\end{abstract}

JEL Classification: G31, G13, D81

Keywords: Real options, trinomial tree, valuation under uncertainty 


\section{Introduction}

Volatility estimation is often the most difficult task in financial option valuation. It is even more challenging with real options, as there is not always a tractable underlying asset with a known process and the volatility does not remain the same during the investment period. Volatility tends to decline over the time during many investment projects as new information and knowledge is gathered. The valuation method applied should also take this into account. On the other hand, a practical valuation method should also be robust and intuitively appealing. Therefore, this paper presents a recombining trinomial lattice for real option valuation (ROV) with changing volatility. The trinomial tree suggested and also its parameterization is straightforward, and as a lattice method, it is also capable to value investments with several interacting parallel and sequential real options.

Contrary to financial options, the underlying asset value in ROV in the beginning is not often a known market-based value but more like an estimate with uncertainty. This is second order uncertainty, or ambiguity, meaning that the underlying asset value is not known well in the beginning. Then, after market information gathering and own activities over time, more reliable estimation of the investment's expected value and its volatility can be made. As a result, volatility tends to decline over the time during many investment projects. For example, knowing the realized product sales for earlier time period is likely to improve the forward-looking estimation of the overall demand.

While the volatility changes with financial options can be considered quit smooth, the situation is often different with real options. Usually the new information arrival, especially in case of R\&D investments, is infrequent, and some of the uncertainty only reveals after own work. Instead of assuming continuous fluctuation according to the geometric Brownian motion, Willner (1995) suggests using pure jump process and Schwartz \& Moon (2000) apply mixed jump-diffusion process. Nevertheless, even a univariate yet time-dependent stochastic process may provide a realistic approach for valuation. Managerial decisions related to the projects usually do not happen continuously but rather at certain time periods. The decisions, i.e. option exercise decisions, are made mostly at certain time points when new information from own activities and markets is gathered and analyzed. As a result, an investment can be considered as staged investment, or like a sequence of call options. More accurate multivariate modeling of the underlying asset value, if even possible, is not therefore necessary between the decision points, as long as the underlying asset value is approximated correctly at the decision point for making optimal decision about option exercise. Because of this rather discrete than continuous approach in decision making, a univariate uncertainty modeling is also able to capture the reality as well. 
Changing volatility with a standard binomial lattice is problematic since the declining volatility means that the tree would not recombine. Without recombining, tree-based real options analysis is impracticable. Guthrie (2009) suggests a modification for binomial tree so that it allows changing volatility. The size of up and down movements and their corresponding transition probabilities are constant throughout the tree, but the time periods are of unequal length. When volatility is high, the time periods are short, so that the state variable changes frequently by the standardized amount. When volatility is lower, the periods are longer so that the changes in the state variable are less frequent. This binomial tree is presented on the left in figure 1.

The method suggested by Guthrie (2009) is sufficiently straightforward extension to the basic CRR binomial tree and as such suitable for practitioners. One shortcoming of the approach is that because of the changing time period lengths, option exercise dates do not necessarily match precisely the actual decision moments. With several different volatility time periods, change in any single volatility during modeling requires adjusting the exercise dates and functions to the correct nodes. The length of times steps needs to be small enough everywhere in a tree for this adjusting to be possible. This is also required so that the transition probabilities would not become negative anywhere in a tree ${ }^{1}$. Another shortcoming is that in case of very small or even non-existing volatility during some time period, any up or down movement deviating from the expected future value - increasing according to the risk-free rate - would make the tree construction impossible.

The trinomial tree presented in this paper is constructed by simultaneously choosing such a parameterization that sets a judicious state space while having sensible transition probabilities between the nodes. The volatility changes are modeled with the changing transition probabilities while the state space of the trinomial tree is regular and has a fixed number of time and underlying asset price levels. This is illustrated on the right side of figure 1, where the width of the arrow in the trinomial tree exemplifies the risk-neutral transition probabilities for up, middle and down movement. In the beginning, when the volatility is higher, the probability of going up or down is larger (thick arrows) than probability of moving to the middle value (thin arrow). Later, when the volatility has diminished, the probability of moving to the middle node is larger (thick arrow) and probabilities for up and down movements (thin arrows) are smaller.

The parameterization presented in this paper for the trinomial tree is an exact solution both to the expected mean value and variance instead of being only an approximation for the variance. Unlike Boyle (1988), the transition probabilities also remain always stable for all dispersion

\footnotetext{
${ }^{1}$ Known issue also with standard (Cox-Ross-Rubinstein 1979) binomial tree
} 
parameter values $\lambda>1$. Also, recombining is set so that $u \cdot d=d \cdot u=m^{2}=e^{2 r \Delta t}$, because otherwise the discretized system would not hold with small or even zero volatility. The trinomial tree is always stable regardless of the length of the time step. Equations to describe stochastic process up and down movements are more accurate even with longer time steps. This is required because the time steps with real option valuation are chosen, due to managerial practicality, to be longer than is commonly used for financial options.
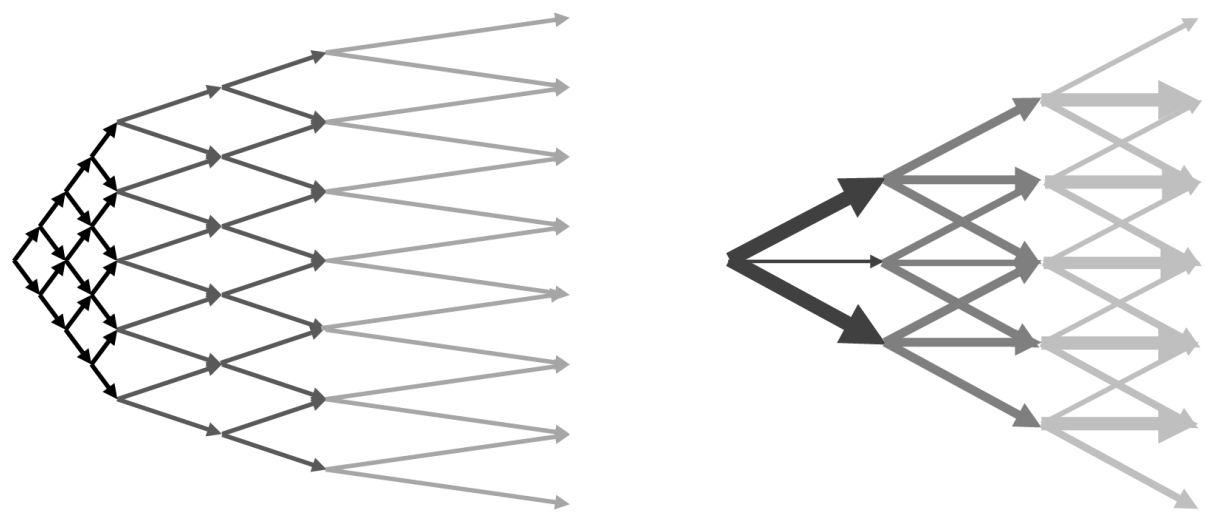

Figure 1: Comparison of Guthrie (2009) binomial tree (left) and the trinomial tree (right) presented in this paper. Thickness of the arrows in the trinomial tree illustrates the transition probabilities between the tree nodes.

This paper also presents a parameterization for the trinomial tree with changing volatility based on cash flow simulation. Therefore this paper also extends research of Copeland \& Antikarov (2001), Herath \& Park (2002), Mun (2003, 2006), Brandão (2005a, 2005b), Godinho (2006), and Haahtela (2008), applying Monte Carlo simulation on cash flows to consolidate a highdimensional stochastic process of several correlated variables into a low-dimension (univariate) geometric Brownian motion process. The volatility parameter $\sigma$ of the underlying asset is then estimated by calculating the standard deviation of the simulated probability distribution for the rate of return. Similarly to Godinho (2006) and Haahtela (2008), cash flow and volatility realizations are conditional on earlier cash flow realizations, and ordinary least squares regression approach is used to estimate the continuation value and its volatility. In contrast with other cash flow simulation based consolidated approaches, the modeling presented in this paper allows changes in volatility while keeping the lattice recombining. Also, while most cash flow simulation based methods commonly assume underlying asset to follow geometric Brownian motion, the modeling and parameterization also allows use of displaced diffusion process of Rubinstein (1983) similarly to Camara (2002), Camara \& Cheung (2004), and Haahtela (2006). 
Next section discusses lattice methods and most common binomial trees. Section 3 extends the theoretical background of lattice methods and discusses trinomial trees. The main contribution of this paper is in Section 4 that describes the construction of the recombining trinomial lattice for real option valuation. Volatility changes are modeled with changing transition probabilities while keeping the state space regular with fixed number of time and underlying asset price levels. Section 5 explains how this trinomial lattice can be parameterized based on simulated cash flow calculation and ordinary least squares regression. Section 6 extends this approach further and shows how to apply displaced diffusion process for the trinomial tree. Section 7 concludes the paper.

\section{Lattice methods}

Lattice models are accurate, robust, and intuitively appealing tools for valuing financial and real options (Hahn 2005, p. 6). Lattices are much more easily explained to and accepted by management because the methodology is much simpler to understand (Mun 2006). This is valuable especially with sequential and parallel compound options, which is often the case in real applications (Trigeorgis 1996, Copeland \& Antikarov 2001). They allow valuation of American options with early exercise possibility and they are suitable for valuing barrier options. Lattice methods also allow valuation of derivatives dependent of several underlying assets (Boyle 1988, Kamrad \& Ritchken 1991) and they can be applied to several stochastic processes, including mean-reverting process (Hahn \& Dyer 2007). Typically lattice methods are of binomial (two states) or trinomial (three states) type, but there are also quadranomial lattices, e.g. for jumpdiffusion process, and pentanomial lattices for rainbow options with two combined and correlated underlying assets (Mun 2006, 306).

Lattice valuation models are based on a simple representation of the evolution of the underlying asset value. The two main ideas with lattice approaches are 1) the modeling of the continuous process with a discrete random walk and 2) the assumption of risk-neutral pricing (Wilmott et al., 1995, 180-181). In the continuous limit, a lattice with an infinite number of time steps to expiration represents a continuous risk-neutral evolution of the asset value. In a lattice method, a tree of possible values of underlying asset prices and their probabilities, given an initial asset price, is built. This tree determines the possible asset prices and the associated probabilities of these asset prices being realized. In other words, a lattice determines the assets prices and probabilities in a state space during each time period over the life-time and at the expiry of the security. The possible values of the security and therefore also the payoff of the option at expiry can then be calculated, and finally, by working back down the tree, the security can be valued. (Wilmott et al., 1995, 182). 
Most simple presentation of a lattice model is a binomial model. A binomial approximation for the geometric Brownian motion process may be developed by assuming that during a short time interval $\Delta t$, stock prices jump from an initial value, $S$, to either up to new value, $S_{u}$, or down to the new value, $S_{d}$. The transition probability of moving up to $S_{u}$ is assumed to be $p$, so that the probability of moving down to $S_{d}$ is $1-p$. These parameters uniquely determine the evolution of the underlying asset, which, in turn, determine a unique value of the option on the stock. (Easton 1996). However, the parameters $p, u, q$, and $d$ cannot be chosen arbitrarily as they must give correct values according to the continuous-time process for the mean and the variance of the change in the stock price during the time interval $\Delta t$. According to Lindeberg's Central Limit Theorem, the following conditions are sufficient to ensure this convergence:

a) Jumps are independent of the stock price level

b) The mean of the binomial distribution is equal to the mean of the lognormal distribution

c) The variance of the binomial distribution is equal to the variance of the lognormal distribution

d) The probabilities $p_{u}$ and $p_{d}$ are positive in the limit between 0 and 1 but not equal either to 0 or 1.

e) The probabilities sum to 1

$$
\begin{aligned}
& p_{u} u+p_{d} d=e^{r \Delta t} \\
& p_{u} u^{2}+p_{d} d^{2}=e^{\left(2 r+\sigma^{2}\right) \Delta t} \\
& p_{u}+p_{d}=1 ; 0<p<1
\end{aligned}
$$

The discretized dynamic process must give correct values to mean, increasing by risk-free interest rate according to the risk-neutral assumption, and variance of the asset dynamics at each time period of length $\Delta \mathrm{t}$. Therefore, Equation (1) must hold for the asset price and Equation (2) for the variance. Equation (3) ensures that the transition probabilities remain between 0 and 1, a necessary condition for the discrete world represented by the tree to preclude arbitrage. Another common restrictions is the recombination condition $u \cdot d=d \cdot u=m^{2}$ so that the binomial lattice branches reconnect at each step. This is an important issue both from a computational efficiency and modeling simplicity perspective, because there are $N+1$ nodes at any stage $N$, whereas there are $2^{\mathrm{N}}$ nodes at the same stage for a non-recombining binomial tree ${ }^{2}$.

\footnotetext{
${ }^{2}$ However, non-recombining binomial trees may be efficient in stage-gate structures common for real options, where only part of the full diffusion process has to be modeled, and numerical accuracy is less important in comparison with financial options.
} 
There are three equations for the four unknowns, $p, u, d$, and $q$. In order to determine these unknowns uniquely we require another equation. Equations (2) and (3) determine all the statistically important properties of the discrete random walk. Therefore, the choice of the fourth equation is somewhat arbitrary (Wilmott et al., 1995). The choices for this additional restriction are theoretically infinite, and there is no obvious criterion to choose among these infinite choices, although it should be ideally selected to achieve the desirable convergence properties of the binomial approximation procedure (Tian 1993). All correctly chosen binomial tree parameterizations represent the same discrete constant volatility world, and all converge to the same theory, i.e the constant-volatility Black-Scholes theory, in the continuous limit. As a result, there are in general an infinite number of (equivalent) binomial trees due to a freedom in the choice of overall growth of the price at tree nodes. If all the node prices of a binomial tree are multiplied by some constant (and reasonably small) growth factor, we will end up with another binomial tree which has different (positive) probabilities but represents the same continuous theory. The familiar CRR (Cox, Ross \& Rubinstein, 1979) binomial tree has the property that all nodes with same spatial index have the same price, making the CRR tree state space look regular in both spatial and temporal directions. Tian (1993) ensures that the third moment of the discrete time process is also correct according to the continuous-time process. The Rendlemann-Bartter (1979) (RB) and Jarrow-Rudd (1979) (JR) binomial trees have the property that all probabilities are equal to $1 / 2$. It is also possible to grow the binomial tree precisely along the forward risk-free interest rate curve so that $u d=e^{2 r \Delta t}$.

Cox, Ross \& Rubinstein (1979) set the fourth equation as $u \cdot d=1$, and given the conditions of (1) - (3), as $\Delta t$ approaches zero, the following equations (4) - (6) hold:

$$
\begin{aligned}
& u=e^{\sigma \sqrt{\Delta t}} \\
& d=e^{-\sigma \sqrt{\Delta t}} \\
& p=\frac{\left(e^{r \Delta t}-d\right)}{(u-d)}
\end{aligned}
$$

CRR is most commonly used binomial model. It consists of a set of nodes, representing possible future stock prices, with a constant logarithmic spacing between these nodes. This spacing is a measure of the future stock price volatility. This leads to a tree with centrality property, meaning that the value of the underlying asset at the central node at time $2 \cdot \mathrm{dt}$ is the same as at time zero. CRR model is intuitive and also pedagogically good (Geske \& Shastri 1985), because it can be 
used in explaining the idea of risk-neutral pricing and delta hedging while also illustrating the discretized stochastic process graphically. Secondly, before the era of PCs and spreadsheet programs, the computations required by the CRR model for options and the Greeks valuation were easier due to the centrality property. Therefore, CRR has become a de facto standard for binomial models yet some other binomial models are better in terms of consistency, accuracy, stability, and convergence (computational) speed.

The parameters suggested by CRR are an exact solution to Equation (1) but only an approximation for Equation (2) For sufficiently small $\Delta t$, (2) can be approximately satisfied. As a result of this approximation, consistency is not perfect, because the variance is slightly downward biased (Trigeorgis 1991). The largest disadvantage of the CRR is that it loses stability if $\Delta t>\sigma^{2} / r^{2}$ and as a result, other probability becomes larger that one and another smaller than zero.

Another way to specify the equations for up and down movements is to set $p_{u}=p_{d}=0.5$. In this case,

$$
\begin{aligned}
& u=e^{\left(r-\sigma^{2} / 2\right) \Delta t+\sigma \sqrt{\Delta t}} \\
& u=e^{\left(r-\sigma^{2} / 2\right) \Delta t-\sigma \sqrt{\Delta t}} \\
& u d=e^{2\left(r-\sigma^{2} / 2\right) \Delta t}
\end{aligned}
$$

As a result, $u \cdot d \neq 1$, and therefore centrality is lost ${ }^{3}$. The advantage of RB parameterization is that it is an exact solution to the equations (1) and (2), and therefore it has perfect consistency so that the mean and variance of the underlying lognormal diffusion process are the same for any step size. Therefore the lattice is always stable, has correct volatility, and converges faster than CRR to the analytical continuous time solution (Jabbour et al., 2001).

There are two small modifications suggested to the previously mentioned common binomial lattice models so that they would become better for real option valuation purposes. The standard deviation of the proportional change in the stock price in a small interval of time $\Delta \mathrm{t}$ is approximately $\sigma \sqrt{\Delta t}$. Therefore, volatility can be interpreted as the standard deviation of the percentage change in the stock price when return is expressed using continuous compounding. Because numerical accuracy requirements are smaller in ROV than with financial options (Mun 2006), most managerially oriented books and their examples suggest using sufficiently long time steps. However, lattice valuation methods assume that $\Delta \mathrm{t}$ is a small time interval, and otherwise

3 Jabbour, Kramin \& Young (2001) present how this can be modeled so that centrality remains as well. 
certain models and their parameterizations become unreliable. Therefore, instead of using $\sigma \sqrt{\Delta t}$, a more precise expression for a deviation over a given time period should be used according ${ }^{4}$ to Equation (10):

$$
\sqrt{e^{\sigma^{2} \Delta t}-1} \approx \sigma \sqrt{\Delta t}
$$

One may also grow the tree along the forward. This can be done by setting $u \cdot d=e^{2 r \Delta t}$. As a result of this centering condition and the previous suggestion for more accurate modeling with longer time steps $\Delta \mathrm{t}$, the binomial tree can be constructed with the following $\mathrm{u}, \mathrm{d}$, and $\mathrm{p}$ according to the Equations (11) - (13):

$$
\begin{aligned}
& u=e^{\sqrt{e^{\sigma^{2} \Delta t}-1}+r \Delta t} \\
& d=e^{-\sqrt{e^{\sigma^{2} \Delta t}-1}+r \Delta t} \\
& p=\frac{\left(e^{r \Delta t}-d\right)}{(u-d)}
\end{aligned}
$$

This tree can be considered either as an extension to the CRR or to the RB parameterization. In this case, both up jump $u$ and down jump $d$ are slightly changed. As a result, the central line follows risk-free rate. Another advantage is that this parameterization is also always stable regardless of the length of the time step $\Delta \mathrm{t}$. Both of these properties are also essential in constructing a robust recombining trinomial tree for changing volatility.

\section{Trinomial trees}

Trinomial trees provide another discrete representation of stock price movement, analogous to binomial trees. The trinomial lattice has three jump parameters $u, m$ and $d$ and three related probabilities parameterized as $p_{1}, p_{2}$, and $p_{3}$. During this time step the stock price can move to one of three nodes: with probability $p_{1}$ to the up node, value $S_{u}$, with probability $p_{2}$ to the middle node, value $S_{m}$, and to the down node, value $S_{d}$, and with probability $p_{3}$. We assume that the probabilities sum to unity, so we set $p_{2}=1-p_{1}-p_{3}$. At the end of the time step, there are five unknown parameters: the two probabilities $p_{1}$ and $p_{3}$, and the three node prices $S_{u}, S_{m}$ and $S_{d}$.

One way to construct trinomial trees is to view two steps of a binomal tree in combination as a single step of a trinomial tree. This can be applied to all standard binomial trees with constant

4 This is based on the properties of the lognormal distribution (Hull 2006, 281-283). Also Jabbour et al. (2001) present this modification. 
volatility, e.g. CRR (1979), JR (1979), RB (1979), Trigeorgis (1991), Tian (1993), and Tian (1999). For example, a two step presentation of the CRR binomial lattice is:

$$
\begin{aligned}
& S_{u}=S e^{\sigma \sqrt{2 \Delta t}} \\
& S_{m}=S \\
& S_{d}=S e^{-\sigma \sqrt{2 \Delta t}} \\
& p_{u}=\left(\frac{e^{r \Delta t / 2}-e^{-\sigma \sqrt{\Delta t / 2}}}{e^{\sigma \Delta t / 2}-e^{-\sigma \sqrt{\Delta t / 2}}}\right)^{2} \\
& p_{d}=\left(\frac{e^{\sigma \sqrt{\Delta t / 2}}-e^{r \Delta t / 2}}{e^{\sigma \Delta t / 2}-e^{-\sigma \sqrt{\Delta t / 2}}}\right)^{2} \\
& p_{m}=1-p_{u}-p_{d}
\end{aligned}
$$

Trinomial trees can also be modeled starting from the same basic assumptions and restrictions that are used for binomial lattices. The transition probabilities are positive in the limit between 0 and 1 and need to sum to unity (20), the mean of the discrete distribution is equal to the mean of the continuous lognormal distribution (21), and the variance is equal to the variance of the continuous distribution (22):

$$
\begin{aligned}
& p_{u}+p_{m}+p_{d}=1 ; \quad 0<\mathrm{p}<1 \\
& p_{u} S u+p_{m} S m+p_{d} S d=S M \\
& p_{u}\left(S^{2} u^{2}-S^{2} M^{2}\right)+p_{m}\left(S^{2} m^{2}-S^{2} M^{2}\right)+p_{d}\left(S^{2} d^{2}-S^{2} M^{2}\right)=S^{2} V .
\end{aligned}
$$

where $M=e^{r \Delta t}$ and $V=e^{\sigma^{2} \Delta t}$.

The first trinomial tree was presented by Boyle (1986). The purpose of this model was to enhance the accuracy and speed over ordinary binomial lattice. Later, Boyle (1988) extended this approach for two state variables. Using equations (20) $-(22)$, and setting $u \cdot d=1$, Boyle solved explicit expressions for transition probabilities:

$$
p_{u}=\frac{\left(V+M^{2}-M\right) u-(M-1)}{(u-1)\left(u^{2}-1\right)}
$$




$$
\begin{aligned}
& p_{d}=\frac{u^{2}\left(V+M^{2}-M\right)-u^{3}(M-1)}{(u-1)\left(u^{2}-1\right)} \\
& p_{m}=1-p_{u}-p_{d}
\end{aligned}
$$

If the original parameters of CRR for $\mathrm{u}$ and $\mathrm{d}$ were used, and setting $\mathrm{m}=1$, some of the transition probabilities would not remain between 0 and 1. Therefore, Boyle suggested using a dispersion parameter $\lambda>1$ to increase $u$ and lower $d$ according to Equations (26) and (27):

$$
\begin{aligned}
& u=e^{\lambda \sigma \sqrt{\Delta \mathrm{t}}} \\
& d=e^{-\lambda \sigma \sqrt{\Delta \mathrm{t}}}
\end{aligned}
$$

where $\lambda$ is greater than 1 . However, this parameterization gives negative transition probabilities with small values of $\lambda$. By trying different values for $\lambda$, a range of values of $u$ is obtained, and there is an interval within this range that produces acceptable values for all the probabilities. Boyle found that for a range of parameter values, the accuracy of the three-jump method with 5 time intervals was comparable to that of the CRR method with 20 time intervals. He also recognized that the best results were obtained when $\lambda$ was set so that the transition probabilities were roughly equal. Kamrad $(1990 ; 14-19)$ enhanced the model to correct the possible problem of negative transition probabilities.

While Boyle (1988) found the optimal solution by trial-and-error experiment so that the probabilities were roughly equal, Tian (1993) and Derman, Kani and Chriss (1996) present equal probability (1/3) trees with two different parameterizations for recombining trinomial tree ${ }^{5}$. Tian (1993) also presented another parameterization based on the idea of matching the first four moments. Tian (1993) found that his two trinomial models converged in practice to the continuous time solution as fast as the model of Boyle (1988). Other possible constructions for trinomial trees are two steps of JR or RB binomial tree which have $\mathrm{p}_{\mathrm{u}}=\mathrm{p}_{\mathrm{d}}=0.25$ and $\mathrm{p}_{\mathrm{m}}=0.5$. Another interpretation and modeling of trinomial lattice is such that $\mathrm{p}_{\mathrm{m}}=2 / 3$ with $u=e^{\sigma \sqrt{3 \Delta t}}$

5 For equal probability (1/3) tree, Tian (1993) has a recombining of $u d=0.5 e^{r \Delta t}\left(3-e^{\sigma^{2} \Delta t}\right)$ while Derman et. al. (1996) has a recombining of $u d=e^{2 r \Delta t-\sigma^{2}}$. Tian (1993) for matching the four moments has a recombining of $u d=e^{r \Delta \mathrm{t}}\left(e^{\sigma^{2} \Delta \mathrm{t}}\right)^{2}$. 
and $d=1 / u$. This parameterization shows the relation of trinomial lattice and explicit finite difference scheme (Hull 2006, 408-409, 427-428). Another common parameterization is to set $\mathrm{p}_{\mathrm{u}}=\mathrm{p}_{\mathrm{d}}=1 / 6$ and $\mathrm{p}_{\mathrm{m}}=2 / 3$ (Derman et al., 1996). As a result, we can construct several kinds of trinomial trees and apply a variety of criteria, all of which may be equally reasonable. However, despite of their limiting similarity, one kind of tree may be more convenient than another.

Trinomial trees have inherently more parameters than binomial trees, so there is more freedom over the choice of the state space. Of the five parameters needed to fix the whole tree, Equations (21) and (22) provide only two constraints, so there are three more parameters than are necessary to satisfy them. These additional parameters can be conveniently used to choose the "state space" of all node prices in the trinomial tree. As a result, it is possible to construct many "economically equivalent" trinomial trees which, in the limit as the time spacing becomes very small, represent the same continuous theory. These properties are often used in valuing implied trees with termand skew structure (Derman et al., 1996) as well as barrier options (Hull 2006, 573-575).

\section{Recombining trinomial tree with changing volatility}

When volatilities are not constant, a common method is to choose the underlying asset prices for each node and then attempt to satisfy the two constraints through the choice of the transition probabilities. This method of initially choosing the state space of prices for the trinomial tree, and then solving for the transition probabilities, is familiar in most applications of the finitedifference method (Derman et al., 1996). We must make a judicious choice of the state space in order to insure that the transition probabilities remain between 0 and 1 , a necessary condition for the discrete world represented by the tree to preclude arbitrage. In choosing a state space, we eliminate three of the five unknown parameters corresponding to the evolution of each node, leaving only the transition probabilities to solve for. In a trinomial tree presented in this paper, construction of the tree happens so that we simultaneously choose such a parameterization that sets a judicious state space, i.e. specify the position of every tree node, while having sensible transition probabilities between the nodes.

As a result, there are only three restricting equations for the three transition probabilities and three jumps, and therefore three additional equations are necessary to define a unique solution. Only one them is quite obvious to ensure that trinomial lattice is recombining: $u \cdot d=m^{2}$. Without recombining, the number of nodes on an $\mathrm{N}$-period trinomial lattice is $\left(3^{\mathrm{N}+1}-1\right) * 0.5$ while with recombining property this reduces to $(\mathrm{N}+1)^{2}$, making such a lattice computationally efficient.

It is convenient to divide the equation (21) by $S$ and (22) by $S^{2}$. Then, Equation (20) can be used to remove $p_{m}$ from (21) and (22). Then, from (21) either $\mathrm{p}_{\mathrm{u}}$ or $\mathrm{p}_{\mathrm{d}}$ can be solved and substituted 
into the latter equation (22). After some simplifications the equations can be solved to give explicit expressions for $\mathrm{p}_{\mathrm{u}}, \mathrm{p}_{\mathrm{m}}$, and $\mathrm{p}_{\mathrm{d}}$ as follows ${ }^{6}$ :

$$
\begin{aligned}
& p_{u}=\frac{\left.M^{2} V-M d-M m+m d\right)}{u^{2}+m d-u m-u d} \\
& p_{d}=p_{u}\left(\frac{m-u}{d-m}\right)+\left(\frac{M-m}{d-m}\right) \\
& p_{m}=1-p_{u}-p_{d}
\end{aligned}
$$

where $M=e^{r \Delta t}$ and $V=e^{\sigma^{2} \Delta t}$. We also set $\mathrm{u} \cdot \mathrm{d}=\mathrm{m}^{2}$ to make the tree recombining. So far we have defined the transition probabilities but not yet the actual up, middle and down jump movements. Derman et al. (1996) state that in a recombining constant volatility trinomial tree ${ }^{7}$, the following equations (31) - (33) must hold:

$$
\begin{aligned}
& S_{u}=S e^{\pi \Delta t+\lambda \sigma \sqrt{\Delta t}} \\
& S_{d}=S e^{\pi \Delta t-\lambda \sigma \sqrt{\Delta t}} \\
& S_{u d}=S_{d u}=S e^{2 \pi \Delta t}
\end{aligned}
$$

for $\lambda>1$ and any reasonable value of $\pi$. Given this knowledge, we start choosing the parameterization to satisfy the requirements and to construct a justified and robust state space.

If the volatility becomes close to zero or is actually zero, the only possibility is that the following move forward in the lattice would have to happen with probability 1 to the expected value of the process in the following time period. As a result, one node value leaving forward from any node value in the state space has to be the expected value of the process in the next time period. Because of the risk-neutrality assumption, the expected value of the underlying asset increases according to the risk-free interest rate. Also, because $\mathrm{u}>\mathrm{m}>\mathrm{d}$, this has to be the middle branch, and as a consequence, we need to set $\mathrm{m}=\mathrm{e}^{\mathrm{r} \Delta \mathrm{t}}$. As a result, in equations (31) $-(33), \pi=\mathrm{r}$.

\footnotetext{
${ }^{6}$ Boyle (1988) has slightly different parameters because he assumed centrality by setting $u \cdot d=1$. This presentation does not have this limiting assumption.

${ }^{7}$ Assuming $\Delta \mathrm{t}$ sufficiently small so that the higher order terms can be ignored
} 
Now we are left with the $\lambda \sigma \sqrt{\Delta t}$ having the dispersion parameter $\lambda>1^{8}$. Previous literature regarding financial options suggests using $\lambda$ values between 1.2 and $3^{0.5}$ (Yuen \& Yang, 2010). Choosing a good value for the $\lambda$ is a balanced decision between a good state space and reasonable transition probabilities. Because of the modified parameterization in comparison with Boyle (1988), there is no need to worry about negative transition probabilities as long as $\lambda>1$.

The smaller the dispersion parameter, the smaller are the up and down jumps. This sets the state space values closer to each other in a vertical level axis. This is desirable, as Widdicks et al. (2002) shows that the option pricing errors with lattice methods are related to the node positioning so that the closer the option exercise price is to the node, the more accurate the approximation of continuous time value. However, when $\lambda$ is close to 1 , the transition probability to jump into the middle branch becomes almost zero. As a result, some values in the state space are hardly reached, and the tree would behave more like a binomial tree. Thus, two advantages of the trinomial tree in comparison with the binomial tree, faster convergence and small oscillation, would be lost, and the precision would reduce.

When $\lambda$ increases, the jump sizes increase as well, but on the other, the transition probabilities become reasonable so that each node in the chosen state space can be reached. Boyle (1988) and Tian (1993) state that the closer the probabilities are to each other, being roughly equal, the faster the tree results converge to the correct value. Setting $\lambda 1.5^{0,5}(\sim 1.2247)$ makes the transition probabilities equal $1 / 3$ when $\Delta$ t gets close to zero ${ }^{9}$. As a result, this can be considered the reasonable upper limit for $\lambda^{10}$. Therefore, the dispersion parameter $\lambda$ should be somewhere between 1 and $1.5^{0.5}$. There is no correct way of setting $\lambda$. However, a justified value for $\lambda$ is 1.12. This makes the state space dense, and provides sufficiently good transition probabilities. If a smaller $\lambda$ would be chosen, the middle node transition probability would start to become quite small. With larger $\lambda$, the up and down jump transition in the first period would become closer to equal, but as a consequence, these transition probabilities would become sufficiently small during other time periods with smaller volatilities.

Another slight modification is to use a more accurate estimate for deviation according to equation (10) instead of $\sigma \sqrt{\Delta t}$. After these modifications, the trinomial lattice parameter construction follows nicely the general form of parameterization for all the transition probabilities and jump sizes $\mathrm{u}, \mathrm{m}$ and $\mathrm{d}$ according to the following equations (34) - (38):

\footnotetext{
${ }^{8}$ Actually, $\lambda$ can also be a dispersion function for which $\lambda>1$ holds.

${ }^{9}$ The probabilities remain sufficiently close to each other even with a small number of times and a long $\Delta \mathrm{t}$.

${ }^{10}$ In case of constructing a trinomial tree as two steps of CRR a tree, it would have $\lambda 2^{0.5}$
} 


$$
\begin{aligned}
& p_{u}=\frac{m^{2}(V-1)}{u^{2}+m d-u m-u d} \\
& p_{d}=p_{u}\left(\frac{m-u}{d-m}\right) \\
& p_{m}=1-p_{u}-p_{d} \\
& u=e^{r \Delta t+\sqrt{e^{(\lambda \sigma)^{2} \Delta t}-1}} \\
& d=e^{r \Delta t-\sqrt{e^{(\lambda \sigma)^{2} \Delta t}-1}}
\end{aligned}
$$

The up (37) and down (38) movements that determine the state space are calculated according to the largest volatility during the investment so that $\sigma=\max \sigma_{\mathrm{i}}$. These values of $\mathrm{u}$ and $\mathrm{d}$ are used for the whole state space during all the time periods regardless of the changing volatility. However, the transition probabilities calculated according to (34) - (36) hold only for the time period with the highest volatility. Transition probabilities for other time periods are calculated so that equation (21) holds for the expected value and (22) for the local volatility.

Having equations (34) - (36) for $\mathrm{p}_{\mathrm{u}}, \mathrm{p}_{\mathrm{d}}$ and $\mathrm{p}_{\mathrm{m}}$ for the time period with highest volatility, we can calculate the transition probabilities $\mathrm{p}_{\mathrm{u}}^{\mathrm{i}}, \mathrm{p}_{\mathrm{u}}^{\mathrm{i}}$ and $\mathrm{p}_{\mathrm{m}}^{\mathrm{i}}$ for other time periods $\mathrm{i}$ according to as:

$$
\begin{aligned}
& p_{u}^{i}=p_{u}\left(\frac{\sigma_{i}}{\sigma_{\max }}\right)^{2} \\
& p_{d}^{i}=p_{d}\left(\frac{\sigma_{i}}{\sigma_{\max }}\right)^{2} \\
& p_{m}^{i}=1-p_{u}^{i}-p_{d}^{i}
\end{aligned}
$$

As a result, we have the parameterization available for constructing a recombining trinomial tree with changing volatility. The use of constructed lattice is similar to ordinary lattice process. After the underlying asset value paths are constructed according to (37) and (38), the option payoff functions are entered into model. Then, the option tree is evaluated by starting at the end of the tree and working backwards with dynamic programming according to the risk-neutral valuation and risk-neutral probabilities (39) - (41). The value of the option $\mathrm{V}_{\mathrm{t}}$ is known at the end at time $T$, with strike price of $I$, and is for a call option worth $V_{t}=\operatorname{Max}\left(S_{t}-I, 0\right)$ and for a put option $V_{t}=\operatorname{Max}\left(I-S_{t}, 0\right)$. Because of the risk-neutrality assumption, the value at each inner node can 
be calculated as the expected value at time $t$ discounted at rate $\mathrm{r}$ for a time period $\mathrm{V}_{\mathrm{t}-1}$ according to:

$$
S_{t-1}=\frac{p_{u} S_{t, u}+p_{m} S_{t, m}+p_{d} S_{t, d}}{e^{r \Delta t}}
$$

Finally, working back through all the nodes provides the value of the option at time zero. The construction of this trinomial tree is illustrated in appendix 1.

\section{Trinomial tree parameterization based on a simulated cash flow calculation}

The volatility of the underlying process might not be known while its standard deviation ${ }^{11}$ may be available. According to the multiplicative geometric Brownian motion, the standard deviation of $\mathrm{S}$ over time is given according to the Equation (43):

$$
\operatorname{std}(S)=S e^{r t} \sqrt{e^{\sigma^{2} t}-1}=S e^{r t} \sqrt{e^{\Sigma \sigma_{i}^{2} t_{i}}-1}
$$

Therefore, if the standard deviations of the underlying asset process at certain time points are known, it is possible to compute the average volatility for each time period. Starting from the beginning of the process, each $\sigma_{\mathrm{i}}$ can be calculated according to Equation (44):

$$
\sigma_{i}=\sqrt{\frac{\ln \left[\left(\frac{s t d(S)_{i}}{S_{0} e^{r t}}\right)^{2}+1\right]-\sum_{i=0}^{i-1}\left(\sigma_{i}^{2} t_{i}\right)}{t_{i}}}
$$

If standard deviation is also unknown, it has to be approximated somehow. Several authors have suggested different variations of applying Monte Carlo simulation on cash flow calculation to estimate the volatility. The existing cash flow simulation based volatility estimation methods are the logarithmic present value approach of Copeland \& Antikarov (2001) and Herath \& Park (2002), conditional logarithmic present value approach of Brandão, Dyer \& Hahn (2005), twolevel simulation and least-squares regression methods of Godinho (2006). All these methods are based on the same basic idea. Monte Carlo simulation on cash flows consolidates a highdimensional stochastic process of several correlated variables into a low-dimension (univariate) geometric Brownian motion summary process. The volatility parameter $\sigma$ of the underlying asset

\footnotetext{
${ }^{11}$ When using cash flow simulation based methods, we can sometimes only observe the standard deviation, and based on that knowledge, determine a justified stochastic process with correct parameter values.
} 
is estimated by calculating the standard deviation of the simulated probability distribution for the rate of return.

The cash flow simulation based volatility estimation presented here is based on using ordinary least squares regression for estimating the underlying asset value $S$ as present value (PV) of all forthcoming cash flows. This stems from the ideas of Carriere (1996) and Longstaff \& Schwartz (2001) regressing the ex post realized payoffs from continuation on functions of the values of the state variables. This regression provides an estimate of the conditional expectation function that determines the optimal exercise strategy between early exercise and continuation in case of American options. Smith (2005), Brandão et al. (2005), Godinho (2006) and Haahtela (2008) have applied this approach so that OLS regression is used for estimating PV with cash flow simulation state variables $X_{k, t}$ as input parameters. However, instead of common approach of directly estimating volatility as standard deviation of rate of return as $z=\ln \left(S_{t+1} / S_{t}\right)$, a regression estimator is constructed to estimate underlying asset value and its standard deviation at different time points. Then, volatility is calculated according to Equation (44). While Longstaff \& Schwartz (2001) approach actually avoids the need to approximate the value process and allows modeling of several kinds of options, it is not as flexible and convenient with several parallel and sequential interacting real options as the consolidated approach presented here.

In the simulation approach for valuing options, a Monte Carlo simulation model is built that takes into account all of the uncertainties in the problem, which can then be used to calculate expected value and volatility for any given moment. To calculate the expected value and volatility at each decision point, we need to examine the expected future cash flows, conditioned on the resolution of all uncertainties up to that time. One alternative to do this is to use regression equations to determine this value and its standard deviation for each time point. Because of the risk-neutrality assumption, knowing $\mathrm{PV}_{\mathrm{t}}$ at any given moment means that we know its expected value at any given moment by discounting or undiscounting the value with risk-free interest rate.. Similarly to previous section, we rather estimate the standard deviation of the stochastic process, and then compute the volatility according to Equation (44).

To do this, we can run a cash flow simulation model and record the PV of the forth-coming cash flows, and the state variable $\mathrm{X}_{\mathrm{k}, \mathrm{t}}$ values for each time period. Then we run a regression relating the PV for each year to the state variables $X_{k, t}$ of the given year and earlier years. The estimated regression equations provide estimates of the expected values as functions conditioned on the resolution of all uncertainties up to each time, and thus, $\mathrm{PV}_{\mathrm{t}}$ is calculated as the expected PV of subsequent cash flows. The expected value is the mean of the PV calculated from the simulated cash flows, but we also need to compute the deviation around the expected value for determining the standard deviation. This is computed as a standard deviation between the $\widehat{P V}_{t}$, calculated as 
the expected PV of subsequent cash flows, and the actual realized $\mathrm{PV}_{\mathrm{t}}$, calculated as the realizations of the subsequent cash flows ${ }^{12}$.

The standard deviation in this approach is actually computed as a standard root mean square of the deviations between the regression estimator results $\widehat{P V}_{t}$ and the realized simulated results $\mathrm{PV}_{\mathrm{t}}$. First, we calculate the estimated expected value for $\widehat{P V}_{t}$ from the values of $\mathrm{X}_{\mathrm{i}, \mathrm{k}}$ generated in that trial using the regression equation. Then, we measure the differences between values predicted by the regression estimator and the values actually observed from the realized simulations. Then we square all the deviations, add them together, divide them by the number of measurements, and take the square root. Because the calculation is based on a forecasting error on simulation based realized values instead of true measured values, term root mean square error is often used instead of standard deviation ${ }^{13}$.

The challenge in this approach is in determining a good regression estimator $\widehat{P V}_{t}$. Usually a linear combination of the most significant cash flow calculation state variables $X_{k, t}$ at the present moment $t$ is a good approximation for the estimator. For the first time period, this expected value estimator can be constructed as follows:

$$
\widehat{P V}_{1}=a_{1}+b_{1,1} X_{1,1}+b_{2,1} X_{2,1}+\cdots+b_{k, 1} X_{k, 1}
$$

On the other hand, it is not necessary to use the cash flow components $\mathrm{X}_{\mathrm{k}, \mathrm{t}}$ of a single year as explanatory variables. Often the whole cash flow of a time period $\mathrm{CF}_{\mathrm{t}}$ may be used as an explaining variable, so that:

$$
\widehat{P V}_{1}=a_{1}+b_{1} C F_{1}
$$

If these models are extended to other time periods, they become:

$$
\widehat{P V}_{t}=a_{t}+b_{1,1} X_{1,1}+\cdots+b_{k, t} X_{k, t}
$$

and

$$
\widehat{P V}_{t}=a_{t}+C F_{1}+\cdots C F_{t-1}+b_{t} C F_{t}
$$

\footnotetext{
12 This approach, similarly to Brandao et al (2005) and Godinho (2006), ensures that only the earlier years' cash flow components are stochastic, and the expected underlying asset is conditional on the earlier outcomes of cash flow simulation.

${ }^{13}$ Standard deviation is often used to describe deviations on realized observable historical data or measurable data. Mathematically, both are practically the same.
} 
A possible combination of the two alternatives is to use them so that the earlier year cash flows $\mathrm{CF}_{1} \ldots \mathrm{CF}_{\mathrm{t}-1}$ and the cash flow calculation components $\mathrm{X}_{\mathrm{k}, \mathrm{t}}$ of the present moment $\mathrm{t}$ are used as explanatory variables as follows:

$$
\begin{aligned}
\widehat{P V}_{t}=a_{t}+C F_{1} & +\cdots C F_{t-1}+b_{1, t} X_{1, t}+\ldots+b_{k, t} X_{k, t} \\
& + \text { possible higher order terms of } X_{t}
\end{aligned}
$$

This approach makes sure that the number of parameters $X_{k, t}$ does not become too large in the estimator. When cash flow calculation model is done correctly, all the future cash flow component values are conditional on earlier realizations. Therefore, the explanatory power of earlier realizations other than their combined value (sum of earlier cash flows CF) is likely not necessary. However, the present state variables $\mathrm{X}_{\mathrm{k}, \mathrm{t}}$ at time $\mathrm{t}$ may provide better explanatory power than using only $\mathrm{CF}_{\mathrm{t}}$. Also, a good possible explanatory regression function for PV may also have variables of higher order and cross-products, non-linear terms, powers, piecewise regressions and other functional forms. These may arise especially if there are cash flow simulation embedded options whose value are based on choosing the optimal decision in each time step during a single cash flow calculation simulation run (Haahtela 2010b). However, structure of the cash flow calculation, regression diagnostics, and sensitivity analysis assist in determining a good forward-looking regression estimator (Haahtela 2010a).

\section{Extending the trinomial tree to follow displaced diffusion process}

One viable and suggested extension to the presented trinomial lattice is to change the commonly assumed geometric Brownian motion into displaced diffusion process suggested by Rubinstein (1983):

$$
d S=\mu S_{\theta} d t+\sigma_{d d}\left(S_{\theta}+\theta\right) d W
$$

Where $\sigma_{d d}$ is volatility of the displaced diffusion and $\theta$ is shifting parameter. The evolution of $S_{T}$ subject to (50) out to a time horizon $\mathrm{T}$ in a risk-neutral world is given by

$$
S_{t}=\left(S_{\theta, 0}+\theta\right) e^{\left(r t+\sigma_{d d} \sqrt{t} z\right)}-\theta \text { with } z \sim N(0,1)
$$

The corresponding underlying asset value distribution is a shifted lognormal distribution. The relationship between volatility $\sigma$ and $\sigma_{\mathrm{dd}}$ is as follows:

$$
S_{0} \sigma=\left(S_{\theta, 0}+\theta\right) \sigma_{d d}
$$

Displaced diffusion process with different values of $S_{\theta}$ and $\theta$ is capable to model stochastic processes that are between multiplicative (lognormal) and additive (arithmetic) processes. It 
provides also good approximation for square root process and also for certain range of constant elasticity of variance processes and extreme value processes. It also allows negative underlying asset values, a property that has been exploited by Camara (2002) and Haahtela (2006).

The modeling of $\sigma_{\mathrm{dd}}$ based on cash flow simulation is done quite similarly as in case of an ordinary geometric Brownian motion. Starting from $\mathrm{S}_{1}$ (estimated as $\mathrm{PV}_{1}$ ), we can fit ${ }^{14}$ the regression estimator values into a shifted lognormal distribution (also called displaced lognormal distribution). This gives us a three-parameter description of the lognormal distribution with parameters $S_{\theta, 1}, \theta_{1}$ and standard deviation of $S_{1}$ in time $t_{1}$. Both $S_{\theta, 1}$ and $\theta_{1}$ are discounted to time $t_{0}$. Then, using $S_{\theta, 0}$ instead of $S_{0}$ in Equation (44), we can calculate $\sigma_{d d, 1}$. Displaced volatilities for other time periods $\sigma_{\mathrm{dd}, \mathrm{t}}$ are calculated using the same equation with the same changes in parameters. Jump sizes $u_{d d}$ and $d_{d d}$ are calculated using $\sigma_{d d}$ and the trinomial tree is constructed using $\mathrm{S}_{\mathrm{dd}, 0}$ as the starting value for the underlying asset stochastic process. Then, from each node value, $\theta_{0} \mathrm{e}^{\text {rt }}$ is subtracted ${ }^{15}$. Another alternative is to subtract $\theta_{0} \mathrm{e}^{\text {rt }}$ only from the terminal values, and then calculate each inner node of earlier time periods according to equation (42). As a result, we have constructed a recombining trinomial tree that allows changes in volatility and also takes into account the level of the underlying asset value.

\section{Conclusions}

This paper presented a recombining trinomial tree for real option valuation with changing volatility. This was done by simultaneously choosing a parameterization that set a judicious state space while having sensible transition probabilities between the nodes. The volatility changes are modeled with the changing transition probabilities while the state space of the trinomial tree is regular and has a fixed number of time and underlying asset price levels.

The presented trinomial lattice can be extended to follow a displaced diffusion process with changing volatility, allowing also taking into account the level of the underlying asset price. The lattice can also be easily parameterized based on a cash flow simulation, using ordinary least squares regression method for changing volatility estimation. The recombining trinomial tree with changing volatility is more flexible and robust for practice use than common lattice models while sustaining their intuitive appeal. Therefore, this approach is also suitable for managerial valuation.

\footnotetext{
${ }^{14}$ All common simulation and distribution fitting software have a shifted lognormal distribution as an alternative. This includes current versions of Palisade's @Risk and Oracle's Crystal Ball MS Excel add-in simulation software.

${ }^{15}$ An easier alternative is to subtract $\theta_{0} e^{\text {rt }}$ only from the end node values, and then calculate each inner node of earlier time periods according to equation (42).
} 


\section{References}

Boyle, P. (1986). Option valuation using a three-jump process. International Options Journal, Vol. 3, 712.

Boyle, P. (1988). A lattice framework for option pricing with two state variables. Journal of Financial and Quantitative Analysis, Vol. 23, pp. 1-12.

Brandao, L., Dyer, J. \& Hahn, W. (2005a). Using Binomial Decision Trees to Solve Real-Option Valuation Problems. Decision Analysis, Vol. 2, No. 2, June 2005, pp. 69-88.

Brandao, L., Dyer, J. \& Hahn, W. (2005b). Response to Comments on Brandão et al. (2005). Decision Analysis, Vol. 2, No. 2, June 2005, pp. 103-109.

Camara, A. (2002). The Valuation of Options on Multiple Operating Cash Flows. 6th Annual Real Options Conference, 4-6 July, Paphos, Cyprus.

Camara, A. \& Chung, S-L. (2006). Option Pricing for the Transformed-Binomial Class, Journal of Futures Markets, Vol. 26, No. 8, pp. 759-787.

Carriere, J. (1996). Valuation of early-exercise price of options using simulations and nonparametric regression. Insurance: Mathematics and Economics. Vol. 19, pp. 19-30.

Copeland, T. \& Antikarov, V. (2001). Real Options: A Practitioner's Guide. Texere.

Cox, J., Ross, S. \& Rubinstein, M. (1979). Option Pricing: A Simplified Approach. Journal of Financial Economics, No. 7, pp. 229-263.

Derman, E., Kani, I., Chriss, N. (1996). Implied Trinomial Trees of the Volatility Smile. Quantitative strategies research notes, Goldman-Sachs, February 1996.

Easton, S. (1996). A note on modified lattice approaches to option pricing. The Journal of Futures Markets. Aug 1996, Vol. 16, No 5. pp. 585-594.

Geske, R. \& Shastri, K. (1985). Valuation by Approximation: A Comparison of Alternative Option Valuation Techniques. Journal of Financial and Quantitative Analysis, Vol. 20, No. 1, pp. 45-71.

Godinho, P. (2006). Monte Carlo Estimation of Project Volatility for Real Options Analysis. Journal of Applied Finance, Vol. 16, No. 1, Spring/Summer 2006.

Guthrie, G. (2009). Learning Options and Binomial Trees. Working paper, Feb. 152009.

Haahtela, T. (2006). Extended Binomial Tree Valuation when the Underlying Asset Distribution is Shifted Lognormal with Higher Moments. 10th Annual International Conference on Real Options, 1417 June, New York, USA. 
Haahtela, T. (2008). Volatility and Ambiguity in Simulation-based Volatility Estimation. 12 ${ }^{\text {th }}$ Annual International Conference on Real Options, 9-12 July, Rio de Janeiro, Brazil.

Haahtela, T. (2010a). Regression sensitivity analysis for cash flow simulation based real option valuation. 6th International Conference on Sensitivity Analysis of Model Output, 19-22 July, Milano, Italy.

Haahtela, T. (2010b). Cash flow simulation embedded real options. International Conference on Applied Operational Research, 25-27 August, Turku, Finland

Hahn, W. (2005). A Discrete-Time Approach for Valuing Real Options with Underlying Mean-Reverting Stochastic Processes. Dissertation, The University of Texas at Austin, USA.

Hahn, W., Dyer, J. (2007). Discrete time modelling of mean-reverting stochastic processes for real option valuation. European journal of operational research. Vol. 184, No. 2, pp. 534-548.

Herath, H.. \& Park, C. (2002). Multi-stage capital investment opportunities as compound real options The Engineering Economist, Vol. 47, No.1, pp. 1-27.

Hull, J. (2006). Options, futures and other derivatives, $6^{\text {th }}$ edition. Prentice-Hall.

Jabbour, G., Kramin, M. \& Young, S. (2001). Two-state Option Pricing: Binomial Models Revisited. Journal of Futures Markets, Nov 2001, Vol. 21, pp. 987-1001.

Jarrow, R. \& Rudd, A. (1982) Approximate option valuation for arbitrary stochastic processes. Journal of Financial Economics, Vol. 10, pp. 347-369.

Kamrad, B. (1990). A multinomial lattice option pricing methodology for valuing risky ventures: Multiple sources of uncertainty. Dissertation, Case Western University, 1990.

Kamrad, B. \& Ritchken, P. (1991). Multinomial Approximating Models for Options with k State Variables. Management Science, Vol. 37, No. 12, pp. 1640-1653.

Longstaff, F. \& Schwartz, E. (2001). Valuing American options by simulation: A simple least-squares approach. Review of Financial Studies, Vol. 14, No. 1, pp. 113-147.

Mun, J. (2003). Real Options Analysis Course: Business Cases and Software Applications. John Wiley \& Sons, New Jersey, USA.

Mun, J. (2006). Real Options Analysis: Tools and Techniques for Valuing Investments and Decisions. John Wiley \& Sons, New Jersey, USA.

Rendleman, R. \& Bartter, B. (1979). Two-state Option Pricing. Journal of Finance, 34, pp. 1092-1110.

Rubinstein, M. (1983). Displaced diffusion option pricing. Journal of Finance, Vol. 38, No.1, March 1983, pp. 213-217. 
Schwartz, E. \& Moon, M. (2000). Evaluating Research and Development Investments, in Project flexibility, agency and competition, eds. Brennan, M. \& Trigeorgis, L., Oxford University Press, pp. 85-106.

Smith, J. (2005) Alternative Approaches for Solving Real-Options Problems (Comments on Brandão et al. 2005). Decision Analysis, Vol. 2, No. 2, June 2005, pp. 89-102.

Tian, Y. (1993). A modified lattice approach to option pricing. The Journal of Futures Markets, Vol. 13, No. 5, pp. 563-577.

Tian, Y. (1999). A flexible binomial option pricing model. The Journal of Futures Markets, Vol. 19, No. 7, pp. 817-843.

Trigeorgis, L. (1991). A Log-Transformed Binomial Numerical Analysis Method for Valuing Complex Multi-Option Investments. Journal of Financial and Quantitative Analysis, Vol. 26, No. 3, pp. 309326.

Trigeorgis, L. (1996). Real Options: Managerial Flexibility and Strategy in Resource Allocation. MIT Press.

Widdicks, M., Andricopoulos, A., Newton, D., Duck, P. (2002). On the enhanced convergence of standard lattice methods for option pricing. Journal of Futures Markets, Vol. 22, No. 4, pp. 315-338.

Willmer, R. (1995). Valuing Start-up venture growth options. in Real options in capital investment: models, strategies, and applications, ed. Trigeorgis, L., Praeger 1995, USA, pp. 221-239.

Wilmott, P., Howison, S., Dewynne, J. (1995). The Mathematics of Financial Derivatives, Cambridge University Press, USA.

Yuen, F., Yang, H. (2010). Option pricing with regime switching by trinomial tree method. Journal of computational and applied mathematics, 233, pp. 1821-1833. 


\section{APPENDIX 1: Illustration of $\mathrm{gBm}$ and displaced diffusion recombining trinomial trees with changing volatility in a spreadsheet format.}

Figure 2 presents a trinomial tree of this paper in a spreadsheet calculation format. The stochastic process is described either according to $\operatorname{Std}(\mathrm{S})_{\mathrm{i}}$ or $\sigma_{\mathrm{i}}$. Other input parameters are $\mathrm{r}, \mathrm{T}, \mathrm{n}$, and $\mathrm{S}$.

If we do not know $\sigma_{i}$, we can calculate them from the standard deviation of the stochastic process according to eq. (44). Then we choose the maximum of $\sigma_{i}$, and together with other parameters, set $\mathrm{m}=\mathrm{e}^{\mathrm{r} \Delta \mathrm{t}}$ and calculate values for $\mathrm{u}$ (eq. 37) and $\mathrm{d}$ (eq. 38). Then, transition probabilities $\mathrm{p}_{\mathrm{u}}, \mathrm{p}_{\mathrm{d}}$ and $\mathrm{p}_{\mathrm{m}}$ are calculated according eq. (34) - (36). Then we calculate the transition probabilities $\mathrm{p}_{\mathrm{u}}^{\mathrm{i}}$,

$\mathrm{p}_{\mathrm{u}}^{\mathrm{i}}$ and $\mathrm{p}_{\mathrm{m}}^{\mathrm{i}}$ for other time periods according eq. (39) - (41). The trinomial tree is constructed and then used for valuation similarly to other common trees.

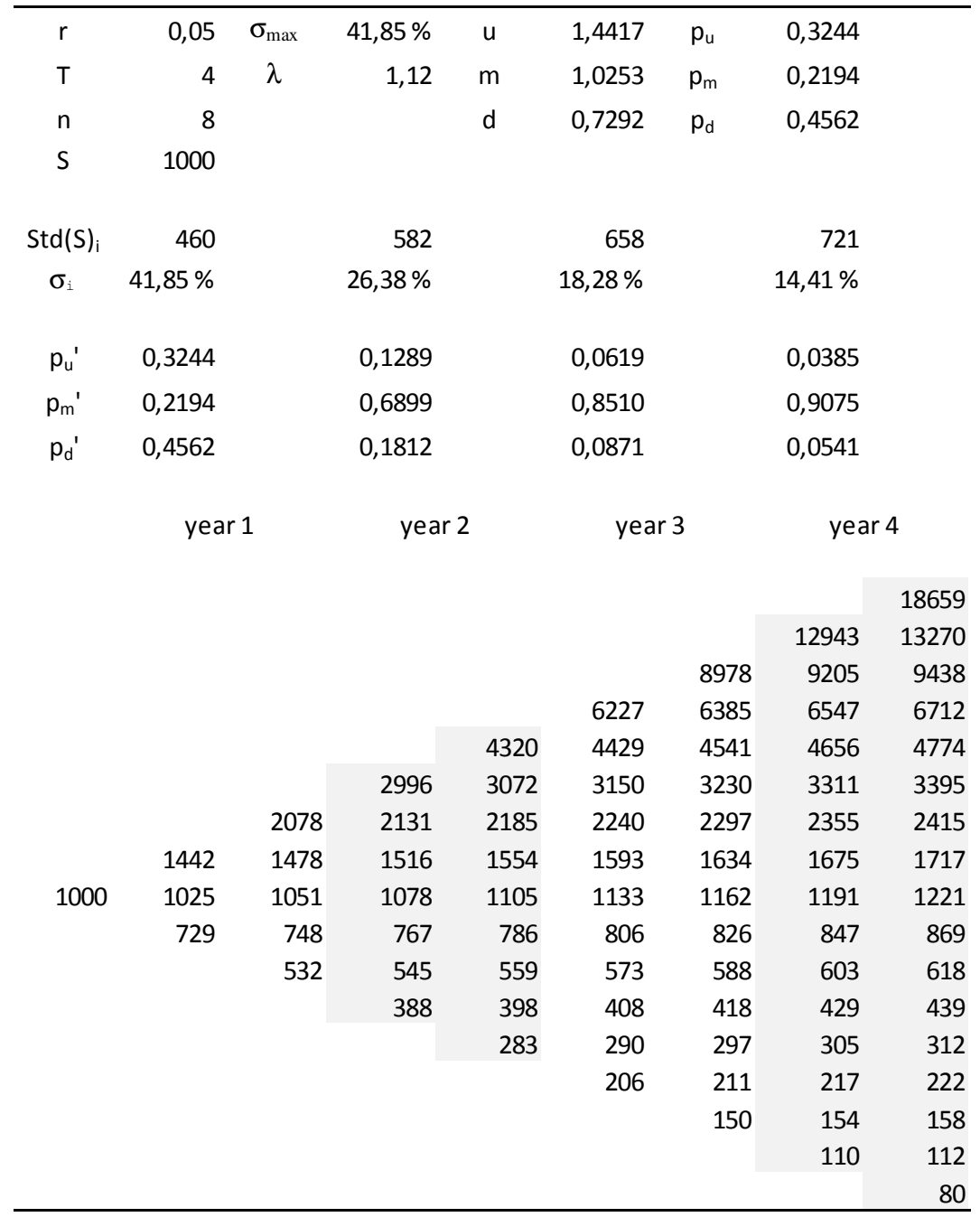

Figure 2: illustration of recombining $\mathrm{gBm}$ trinomial tree with changing volatility in a spreadsheet. 
The following figure 3 presents the trinomial tree with displaced diffusion process. Both trees (gBm and displaced diffusion) have the same expected value and standard deviation, but different shape for the uncertainty cone. Displaced diffusion process also allows negative underlying asset values. Similarly to the $\mathrm{gBm}$ tree, the middle branch value always increases according to $m=e^{r \Delta t}$.

From the spreadsheet modeling perspective, the main difference is that $\mathrm{u}_{\mathrm{dd}}$ and $\mathrm{d}_{\mathrm{dd}}$ are calculated using $\sigma_{\mathrm{dd}, \max }$. Also, tree is constructed using $S_{\mathrm{dd}, 0}$ as the starting node value, and $\theta_{0} \mathrm{e}^{\mathrm{rt}}$ is subtracted from each node, or only from the end node values, and then the earlier node values of the tree are calculated according to eq. (42).

\begin{tabular}{|c|c|c|c|c|c|c|c|c|}
\hline$r$ & 0,05 & $\sigma_{\mathrm{dd}, \max }$ & $21,62 \%$ & $u_{d d}$ & 1,2184 & $\mathrm{p}_{\mathrm{u}}$ & \multicolumn{2}{|l|}{0,3623} \\
\hline $\mathrm{T}$ & 4 & $\lambda$ & 1,12 & $\mathrm{~m}$ & 1,0253 & $\mathrm{p}_{\mathrm{m}}$ & \multicolumn{2}{|l|}{0,2072} \\
\hline $\mathrm{n}$ & 8 & & & $d_{d d}$ & 0,8629 & $\mathrm{p}_{\mathrm{d}}$ & \multicolumn{2}{|l|}{0,4305} \\
\hline \multirow[t]{2}{*}{$S_{d d, 0}$} & 2000 & $\theta$ & -1000 & & & & & \\
\hline & \multicolumn{2}{|c|}{ year 1} & \multicolumn{2}{|c|}{ year 2} & \multicolumn{2}{|c|}{ year 3} & \multicolumn{2}{|c|}{ year 4} \\
\hline $\operatorname{Std}(S)_{i}$ & \multicolumn{2}{|c|}{460} & \multicolumn{2}{|c|}{582} & \multicolumn{2}{|c|}{658} & \multicolumn{2}{|c|}{721} \\
\hline$\sigma_{d d, i}$ & \multicolumn{2}{|c|}{$21,62 \%$} & \multicolumn{2}{|c|}{$14,24 \%$} & \multicolumn{2}{|c|}{$10,05 \%$} & \multicolumn{2}{|c|}{$8,00 \%$} \\
\hline$\sigma_{i}$ & \multicolumn{2}{|c|}{$41,85 \%$} & \multicolumn{2}{|c|}{$26,38 \%$} & \multicolumn{2}{|c|}{$18,28 \%$} & \multicolumn{2}{|c|}{$14,41 \%$} \\
\hline $\mathrm{p}_{\mathrm{u}}{ }^{\prime}$ & \multicolumn{2}{|c|}{0,3623} & \multicolumn{2}{|c|}{0,1571} & \multicolumn{2}{|c|}{0,0783} & \multicolumn{2}{|c|}{0,0495} \\
\hline$p_{m}{ }^{\prime}$ & \multicolumn{2}{|c|}{0,2072} & \multicolumn{2}{|c|}{0,6561} & \multicolumn{2}{|c|}{0,8287} & \multicolumn{2}{|c|}{0,8916} \\
\hline \multirow[t]{9}{*}{$\mathrm{p}_{\mathrm{d}}^{\prime}$} & \multicolumn{2}{|c|}{0,4305} & \multicolumn{2}{|c|}{0,1867} & 0,09 & & 0,05 & \\
\hline & & & & & & & & 8489 \\
\hline & & & & & & & 6779 & 6951 \\
\hline & & & & & & 5380 & 5516 & 5656 \\
\hline & & & & & 4236 & 4343 & 4453 & 4566 \\
\hline & & & & 3302 & 3385 & 3471 & 3559 & 3649 \\
\hline & & & 2539 & 2604 & 2669 & 2737 & 2806 & 2877 \\
\hline & & 1918 & 1966 & 2016 & 2067 & 2119 & 2173 & 2228 \\
\hline & 1411 & 1447 & 1484 & 1521 & 1560 & 1599 & 1640 & 1681 \\
\hline 1000 & 1025 & 1051 & 1078 & 1105 & 1133 & 1162 & 1191 & 1221 \\
\hline & 700 & 718 & 736 & 755 & 774 & 794 & 814 & 834 \\
\hline & & 438 & 449 & 460 & 472 & 484 & 496 & 509 \\
\hline & & & 207 & 212 & 218 & 223 & 229 & 234 \\
\hline & & & & 3 & 4 & 4 & 4 & 4 \\
\hline & & & & & -177 & -181 & -186 & -190 \\
\hline & & & & & & -336 & -345 & -354 \\
\hline & & & & & & & -479 & -491 \\
\hline & & & & & & & & -607 \\
\hline
\end{tabular}

Figure 3: Illustration of recombining displaced diffusion trinomial tree with changing volatility. 